Research Article

\title{
Cooperative Midcourse Guidance Law with Communication Delay
}

\author{
Zihao Wu $\mathbb{D}^{1},{ }^{1}$ Quanbin Ren, ${ }^{1}$ Zhiqing Luo, ${ }^{2}$ Yangwang Fang $\mathbb{D}^{3},{ }^{3}$ and Wenxing Fu $\mathbb{D}^{3}$ \\ ${ }^{1}$ Aerospace Institute, Northwestern Polytechnical University, Xi'an, China \\ ${ }^{2}$ Academy of Aerospace Solid Propulsion Technology, Xi'an, China \\ ${ }^{3}$ Unmanned Systems Research Institute, Northwestern Polytechnical University, Xi'an, China
}

Correspondence should be addressed to Wenxing Fu; wenxingfu@nwpu.edu.cn

Received 15 July 2021; Accepted 17 August 2021; Published 11 September 2021

Academic Editor: Shaoming $\mathrm{He}$

Copyright (c) 2021 Zihao Wu et al. This is an open access article distributed under the Creative Commons Attribution License, which permits unrestricted use, distribution, and reproduction in any medium, provided the original work is properly cited.

The initial conditions of the cooperative terminal guidance law, which are the terminal conditions of the cooperative midcourse guidance, have a greater impact on its cooperation and guidance precision, so it is worthy of investigating the cooperative midcourse guidance. In addition, the problem of communication delay between the network nodes is inevitable and has a greater impact on the cooperative guidance law. To solve the above problems, a novel distributed cooperative midcourse guidance (DCMG) law with communication delay is proposed by combining the cooperative term with a distributed consensus protocol including communication delay under the directed communication topology. Firstly, a DCMG law with communication delay is designed by combining the trajectory shaping guidance with the distributed protocol including communication delay under the directed communication topology; secondly, the consensus of the proposed DCMG law with communication delay under the directed graph is proved; finally, the effectiveness and superiority of the proposed DCMG law are verified by numerical simulations.

\section{Introduction}

As more and more high-value targets are equipped with antimissile systems, the traditional single missile's killing ability of attacking a single target has seriously deteriorated. Therefore, a multimissile cooperative attacking strategy is effective for increasing the killing possibility. For mediumand long-range missiles, the cooperative midcourse guidance phase needs to provide good initial conditions for the cooperative terminal guidance phase, ensuring that the cooperative terminal guidance law can complete the task of hitting the target at the same time within a very limited time. During the last few decades, cooperative guidance has attracted much more attention.

In the early studying stages of cooperative guidance, some scholars designed the impact time control guidance (ITCG) law to realize the salvo strike through an appropriate predesigned impact time for all missiles, which can ensure all missiles arriving at the target simultaneously. For a stationary target, Jeon et al. introduced an ITCG law which can make each missile of the group hit the target individually at the same time [1]. Lee et al. presented an ITCG guidance considering both the time and angle constrain [2]. The authors in [3] designed a cooperative guidance law based on the missile's acceleration. However, the predesigned impact time has a greater impact on the ITCG law. If the predesigned impact time is inappropriate, the missiles cannot reach the target simultaneously due to the limitation of the missile speed and maneuverability. Because no actual cooperative operation is conducted in real time, so the ITCG guidance cannot be viewed as a real-time multimissile cooperative guidance.

Subsequently, with the development of communication networks, the cooperative homing guidance based on communication has attracted much more attention, in which multimissiles attack a target simultaneously by interacting with the cooperative information in the formation [4]. Based on the estimated time-to-go, the authors in $[5,6]$ introduced cooperative guidance laws to achieve consensus. Under time-varying communication topology, Wang et al. [7] presented a guidance with angle constraint in a leader-following 
coordination strategy. Zhou and Yang [8] designed a cooperative guidance law for moving targets. Furthermore, they used the missile's range-to-go as a cooperative parameter to design a cooperative guidance [9]. Based on finite time theory, the authors in [10-12] designed distributed cooperative guidance laws, which can realize finite time consensus. Zhao et al. in [13] combined time-to-go estimation with a cooperative term to design three-dimensional cooperative guidance based on traditional PNG, which is easily implemented. Later, Zhao and Zhou used the receding horizon control technique to design a guidance against a stationary target [14]. The authors in [15] introduced cooperative guidance laws based on the direction and normal direction of LOS considering impact-angle constraint to achieve consensus in finite time. Based on the direction and normal direction of LOS, Zhang and Yang [16] provide a fully distributed, adaptive, and optimal approach to address the problem of simultaneous attack against a maneuvering target with multiple missiles. Furthermore, they designed a cooperative guidance based on a time-varying terminal sliding mode for maneuvering target [17]. Lyu et al. [18] proposed a three-dimensional finite-time cooperative guidance for multiple missiles without radial velocity measurements. Based on the ITCG guidance theory, the designed cooperative guidance law in [19] requires each missile to communicate with other missiles; that is, communication topology must to be strongly connected. The authors in $[20,21]$ designed distributed cooperative guidance considering the impact-angle constraint, which can ensure all missiles attack the target at a desired angle simultaneously. Zhang et al. used a time-varying navigation parameter with traditional PNG to design the cooperative guidance [22]. Based on fixed time theory, Chen et al. provided a fixed-time robust cooperative guidance [23]. Chen et al. [24] designed a cooperative guidance for multiple powered missiles. In view of the characteristics of the communication topology, Zhou et al. proposed cooperative guidance laws in discrete-time communication and sampled data communication, respectively $[25,26]$.

The above results are applicable to the cooperative terminal guidance phase, which can ensure that all missiles hit targets at the same time under the directed graph, and the differences of relative ranges and times-to-go can be zero. However, if the initial conditions of the cooperative terminal guidance for medium-range and long-range missiles do not meet the requirements, the final cooperative strike task cannot be completed. For example, the relative range from the target and the time-to-go of one missile are $10 \mathrm{~km}$ and $20 \mathrm{~s}$, respectively, and the relative range from target and the time-to-go of another missile are $20 \mathrm{~km}$ and $35 \mathrm{~s}$, respectively. Under this circumstance, it is difficult for cooperative terminal guidance law to guarantee the completion of the task of hitting the target at the same time within the limited fight time. Therefore, cooperation in the midcourse guidance phase is very important and provides good initial conditions for the cooperative terminal guidance phase. The cooperative midcourse guidance (CMG) law is an effective way to ensure the initial conditions of the terminal guidance phase to be satisfied. Based on the Dubins path planning method, Zeng et al. [27] designed a cooperative strategy to synchronize the arrival time of the missiles in the midcourse guidance phase with a distinct leaderfollowing framework, but the missile needs to fly around a circular trajectory, which consumes a lot of energy. In [28], $\mathrm{Wu}$ et al. presented a CMG law to realize a cooperative task, but suitable for centralized network. However, none of the above research results consider the influence of communication delay on the cooperative guidance law.

In the real battlefield environment, communication delay is inevitable and has a greater impact on the distributed cooperative guidance law. He et al. introduced a three-dimensional cooperative guidance law with communication delay [29]. Liu et al. proposed a cooperative guidance law with communication delay for a moving target [30]. Zhaohui et al. introduced a fixed-time cooperative guidance law with input delay for a moving target [31]. Zhang et al. presented a cooperative guidance law with communication delay based on the time-to-go [32]. The above studies all aim at undirected communication topology structure which requires two nodes to communicate with each other. Compared with the undirected graph, the directed graph requires a directed path between two nodes, which is more suitable for practical engineering. The authors in [30,33] proposed cooperative guidance laws with communication delay based on the directed graph and obtained the upper bound of communication delay through the linear matrix inequalities (LMI) approach, but the LMI approach is more complicated and may not have a solution. It should be noted that the above research results are all based on the cooperative terminal guidance phase with communication delay and did not pay attention to the cooperative midcourse guidance phase. Therefore, this paper, in the case of cooperative midcourse guidance phase with communication delay, proposes a cooperative midcourse guidance law with communication delay to overcome the influence of communication delay. Furthermore, the LMI method is not convenient to apply in the engineering practice, so it is necessary to study a method that can easily obtain the upper bound of the communication delay.

Based on the above analysis, the distributed cooperative midcourse cooperative guidance law based on the directed graph with communication delay is worth studying, and the upper bound of the communication delay can be easily obtained to facilitate practical applications. Therefore, a novel DCMG law with communication delay is investigated under the directed graph in this paper. The main contributions of this paper are summarized as follows: (A) Compared with Refs. [29-32], this paper proposes a DCMG law with communication delay against a maneuvering target under the directed graph in the three-dimensional planes. (B) Compared with Refs. [29-31], this paper solves the communication delay problem under the directed communication topology and has more practical engineering significance than the undirected graph. (C) Refs. [30, 33] need to solve the LMI in real time to obtain the upper bound of the communication delay, which places higher requirements on the computing power of the system. The proposed guidance law can directly give the maximum communication delay through the communication topology, which is more conducive to engineering realization. 
The remainder of this paper is organized as follows: a few preliminaries and some conclusions are introduced in Section 2. In Section 3, the proposed DCMG law with communication delay is proposed, and its time-space consensus under the directed communication topology is proved, and the upper bound of the communication delay is given explicitly. Simulation results are given in Section 4. Finally, the conclusions are drawn in Section 5.

\section{Preliminaries}

In this section, a few preliminaries are given. Some definitions and important conclusions are presented, and the investigation goal is introduced.

2.1. Graph Theories. Suppose the graph $G$ with $n$ nodes of missile is the communication topology of the multimissile system, which consists of a pair $(V, E)$, where $V=\left\{v_{1}, v_{2}\right.$, $\left.\cdots, v_{n}\right\}$ represents the set of nodes, and $E \in V \times V$ indicates edges between nodes. An edge $\left(v_{i}, v_{j}\right)$ presents that the $i$ th node is connected with the $j$ th node. The adjacency matrix $A=\left(a_{i j}\right) \in R^{n \times n}$ is defined as $a_{i j}=1$ if $\left(v_{i}, v_{j}\right) \in E$ and 0 otherwise. If there exists at least one node having a directed path to all other nodes, then the directed graph is said to include a directed spanning tree. The Laplacian matrix of $G$ is $L=\left(l_{i j}\right.$ ) $\in R^{n \times n}$, where

$$
l_{i j}= \begin{cases}\sum_{k=1, k=i}^{n} a_{i k}, & j=i, \\ -a_{i j}, & j \neq i .\end{cases}
$$

Obviously, the matrix $L=\left(l_{i j}\right) \in R^{n \times n}$ has one zero eigenvalue $\lambda_{1}(L)=0$, and all the other eigenvalues $\lambda_{i}(L)(i=2$, $\cdots, n)$ are positive. In this paper, the following assumption is made about the communication topology.

Assumption 1. The directed graph $G$ has a directed spanning tree.

2.2. Problem Formulation. In this part, the threedimensional geometry between several missiles and a single maneuvering target is considered, and some assumptions are presented as follows.

Assumption 2. Missiles and target are considered the mass point in space.

The engagement geometry in three-dimensional planes is depicted in Figure 1, where $\left(X_{I}, Y_{I}, Z_{I}\right)$ denotes the inertial system; $V_{i}$ and $V_{T}$ indicate the speeds of the $i$ th missile and target, respectively; and $M_{i}$ and $T$ indicate the $i$ th missile and target, respectively.

In order to ensure the attack effect, the DCMG law is required to provide better initial conditions for cooperative terminal guidance law. The seeker of the missile cannot work in the midcourse guidance phase, so the missile cannot detect and track the target directly, but through external detector, the missile still receive the location and velocity

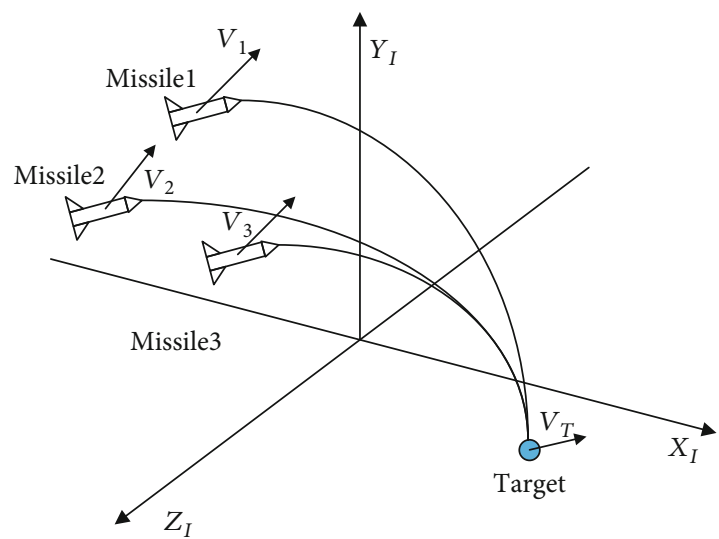

FIGURE 1: Engagement geometry in a 3-D plane for a single missile.

information of itself and the target, as well as the estimated acceleration information of the target obtained through observation and estimation. Therefore, based on this information, which can be directly obtained in the midguidance phase, we will design the DCMG law in the inertial system. Suppose the dynamics of the missile are given as

$$
\begin{aligned}
& \left\{\begin{array}{l}
\dot{x}_{m i}(t)=V_{x i}(t), \\
\dot{y}_{m i}(t)=V_{y i}(t), \\
\dot{z}_{m i}(t)=V_{z i}(t),
\end{array}\right. \\
& \left\{\begin{array}{l}
\dot{V}_{x i}(t)=a_{x i}(t), \\
\dot{V}_{y i}(t)=a_{y i}(t), \\
\dot{V}_{z i}(t)=a_{z i}(t),
\end{array}\right.
\end{aligned}
$$

where $\left(x_{m i}, y_{m i}, z_{m i}\right),\left(V_{x i}, V_{y i}, V_{z i}\right)$, and $\left(a_{x i}, a_{y i}, a_{z i}\right)$ indicate the position, velocity, and acceleration of the $i$ th missile in the inertial system, respectively.

In order to ensure the consistency of the DCMG law, the design of the communication topology plays a crucial role. Compared with the undirected graph, the directed graph can effectively save communication resources, so it is more suitable for practical application. In addition, the communication delay cannot be ignored. Taking into account the above factors, this paper designs a DCMG law with communication delay to ensure that the requirements of cooperative terminal guidance are met. In the cooperative terminal guidance phase, the goal of the cooperative terminal guidance law is to ensure that all missiles reach the target at the same time, that is,

$$
\begin{gathered}
\lim _{t \longrightarrow t_{f}}\left(R_{i}(t)-R_{j}(t)\right)=0, \\
\lim _{t \longrightarrow t_{f}}\left(t_{g o i}(t)-t_{g o j}(t)\right)=0,
\end{gathered}
$$

where $R_{i}(t)$ presents the relative range between the target and the $i$ th missile, $t_{g o i}$ indicates the time-to-go of the $i$ th missile, and $t_{f}$ represents the end of the terminal guidance 


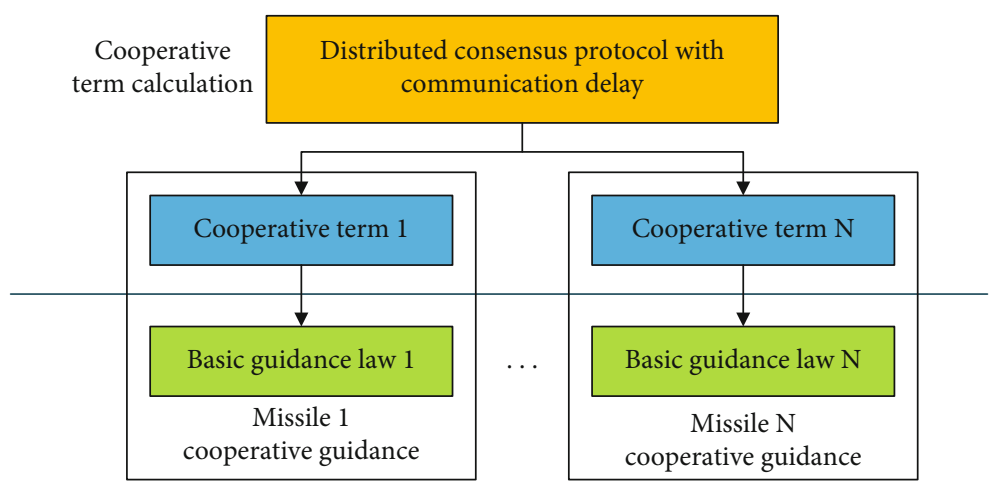

Figure 2: The scheme of the DCMG law with communication delay.

phase. If the differences of $R_{i}(t)$ and $t_{g o i}(t)$ of missiles are too large at the beginning of the terminal guidance phase, the cooperative terminal guidance law cannot ensure that all missiles reach the target at the same time in a limited time. Therefore, in order to ensure the effectiveness of the cooperative terminal guidance law, the differences of the $R_{i}$ $(t)$ and $t_{g o i}(t)$ of missiles should not be too large at the end of cooperative midcourse guidance phase. Based on the above analysis, the cooperative guidance law must meet the following conditions, that is,

$$
\begin{gathered}
\lim _{t \longrightarrow t_{m f}}\left(R_{i}(t)-R_{j}(t)\right)<\sigma_{1}, \\
\lim _{t \longrightarrow t_{m f}}\left(t_{g o i}(t)-t_{g o j}(t)\right)<\sigma_{2},
\end{gathered}
$$

where $t_{m f}$ represents the end of the cooperative mid-course guidance phase and $\sigma_{1}$ and $\sigma_{2}$ are positive constants.

\section{Guidance Law Design}

In this section, the DCMG law with communication delay is presented and proof of consensus is given.

3.1. Guidance Law Scheme. According to the Figure 2, the DCMG law with communication delay mainly consists of the basic guidance law and the cooperative term based on the distributed consensus protocol with communication delay, which can be expressed as

$$
a_{i}(t)=a_{b i}(t)+a_{c i}(t-\tau),
$$

where $a_{b i}(t)$ and $a_{c i}(t-\tau)$ represent the basic guidance law and the cooperative term with communication delay, respectively. Considering the limitation of the type of information, the accelerations are designed based on the $X, Y$, and $Z$ axes in the inertial coordinate system. In order to provide the better initial conditions for the terminal guidance phase, we take the location of the virtual target collision points as cooperative parameters, which are gradually becoming consistent through the multiagent consistency protocol with communication delay. In the midcourse guidance phase, due to the impact of the cooperative terms, the proposed DCMG law with communication delay can ensure that the
$R_{i}(t)$ and $t_{g o i}(t)$ are consistent when the terminal guidance phase begins. Due to the limited space, this paper only discusses the acceleration design of the $X$ axis, and the same applies to the $Y$ axis and the $Z$ axis.

3.2. Guidance Law Design. In the midcourse guidance phase, we use the virtual target collision point as the final target point. The $x_{m i}^{d}(t)$ represents the position of virtual target collision point corresponding to the $i$ th missile and can be expressed as

$$
x_{m i}^{d}(t)=x_{T}(t)+V_{T x}(t) t_{g o i}(t),
$$

where $x_{T}(t)$ represents the position of the target and $V_{T x}(t)$ denotes the speed of the target. Inspired by the trajectory shaping guidance law in $[28,34]$, the acceleration of the $i$ th missile can be given as

$$
\begin{aligned}
a_{i}(t)= & \frac{6\left(x_{m i}^{d}(t)-x_{m i}(t)+\left(V_{T x}(t)-V_{x i}(t)\right) t_{g o i}(t)\right)}{t_{g o i}^{2}(t)} \\
& +\frac{V_{T x}\left(t_{f}\right)-V_{x i}\left(t_{f}\right)-\left(V_{T x}(t)-V_{x i}(t)\right)}{t_{g o i}(t)} \\
& +a_{T x}(t)-\left[P_{i}(t)\left(x_{\mathrm{mi}}^{d}(t)-\bar{x}_{m i}{ }^{d}(t)\right)\right],
\end{aligned}
$$

$P_{i}(t)=\frac{p_{i} \operatorname{sgn}\left|t_{g o i}(t)-\bar{t}_{g o i}(t)\right| \operatorname{sgn}\left|2\left(x_{m i}(t)-x_{T}(t)-V_{T x}(t) \bar{t}_{g o i}(t)\right)\right|}{V_{T x}(t)\left(x_{T}(t)-x_{m i}(t)\right)}$,

where $V_{T x}\left(t_{f}\right)$ and $V_{x i}\left(t_{f}\right)$ present the terminal velocities of the target and the missile, respectively; $x_{m i}(t)$ indicates the position of the $i$ th missile; $a_{T x}(t)$ indicates the acceleration of the target; $P_{i}(t)$ is a cooperative coefficient for the cooperative term; $p_{i}$ is a coefficient to be determined; and $t_{g o i}(t)$ can be expressed as

$$
t_{g o i}(t)=-\frac{R_{i}(t)}{\dot{R}_{i}(t)} .
$$


$\bar{x}_{m i}^{d}(t)$ is the cooperative parameter expressed as

$$
\bar{x}_{m i}^{d}(t)=\frac{1}{N_{i}\left(j \mid j \in N_{i}(t)\right)} \sum_{j \in N_{i}(t)} x_{m j}^{d}(t),
$$

where $N_{i}(t)$ presents the number of the $i$ th node and its neighbor.

Remark 3. When the missile is in the mid-course guidance phase, it is considered that the angle between the speed of the missile and the line of sight of the missile is very small, so the time-to-go estimation as described by Equation (9) is feasible for midguidance guidance phase. In addition, the cooperative guidance law based on Equation (9) in Refs. $[12,15,18]$ can also effectively complete the task of striking maneuvering targets at the same time.

From the Equation (7), the cooperative term $P_{i}(t)\left(x_{\mathrm{mi}}^{d}\right.$ $\left.(t)-\bar{x}_{m i}{ }^{d}(t)\right)$ ensures that $x_{\mathrm{mi}}^{d}(t)$ converges to $\bar{x}_{m i}{ }^{d}(t)$ (the proof is given in Section 3.3). However, each node in the distributed communication topology cannot receive the $x_{m i}^{d}(t)$ of all other nodes, so $\bar{x}_{m i}{ }^{d}(t)$ of Equation (8) cannot be ensured to converge to a unified state. Furthermore, under the distributed communication network, there exists a communication delay in the process of cooperative information transmission. Therefore, in order to ensure the convergence of $\bar{x}_{m i}{ }^{d}(t)$ in the presence of communication delay, the consistency protocol about the state $\bar{x}_{m i}{ }^{d}(t)$ based on the multiagent consistency protocol with communication delay can be given as

$$
\left\{\begin{array}{l}
\dot{\bar{x}}_{m i}{ }^{d}(t)=u_{i}(t), \\
u_{i}(t)=-\sum_{j=1}^{N_{i}} a_{i j}\left[\bar{x}_{m i}{ }^{d}(t-\tau)-\bar{x}_{m j}{ }^{d}(t-\tau)\right], \quad i=1,2, \cdots, n,
\end{array}\right.
$$

where $a_{i j}$ represents the $(i, j)$ element in the adjacency matrix and $\tau$ indicates communication delay. Combining Equations (7) and (8) with (11), we can obtain DCMG law with communication delay as

$$
\left\{\begin{array}{l}
a_{i}(t)=\frac{6\left(x_{m i}^{d}(t)-x_{m i}(t)+\left(V_{T x}(t)-V_{x i}(t)\right) t_{g o i}(t)\right)}{t_{g o i}^{2}(t)}+\frac{V_{T x}\left(t_{f}\right)-V_{x i}\left(t_{f}\right)-\left(V_{T x}(t)-V_{x i}(t)\right)}{t_{g o i}(t)}+a_{T x}(t)-\left[P_{i}(t)\left(x_{m i}^{d}(t)-\bar{x}_{m i}{ }^{d}(t)\right)\right] \\
\dot{\bar{x}}_{m i}{ }^{d}(t)=-\sum_{j=1}^{N_{i}} a_{i j}\left[\bar{x}_{m i}{ }^{d}(t-\tau)-\bar{x}_{m j}{ }^{d}(t-\tau)\right] .
\end{array}\right.
$$

3.3. Guidance Consistency. When the cooperative term $\left(x_{m i}^{d}\right.$ $\left.(t)-\bar{x}_{m i}{ }^{d}(t)\right)$ converges to 0 with $t \longrightarrow \infty$, Equation (7) can ensure the $i$ th missile and its neighbor arriving at the handover area simultaneously, that is,

$$
\lim _{t \longrightarrow+\infty}\left(x_{m i}^{d}(t)-\bar{x}_{m i}^{d}(t)\right)=0
$$

In the following, the convergence conditions for $x_{m i}{ }^{d}(t)$ $\longrightarrow \bar{x}_{m i}{ }^{d}(t)$ are given.

Theorem 4. Suppose that there exists a directed spanning tree in graph $G, V_{T x}(t)>0$, and $p_{i}>0$. Under the proposed DCMG law (12), $x_{m i}{ }^{d}(t) \longrightarrow \bar{x}_{m i}{ }^{d}(t)$ when $t \longrightarrow \infty$.

Proof. Define the squared difference between $x_{m i}^{d}(t)$ and $\bar{x}_{m i}{ }^{d}(t)$ as

$$
\eta_{i}(t)=\left(x_{m i}^{d}(t)-\bar{x}_{m i}^{d}(t)\right)^{2}
$$

Based on the Taylor series, we can get equations as follows:

$$
\begin{aligned}
& x_{m i}{ }^{d}(t+\Delta t)=x_{m i}(t)+V_{x i}(t) \Delta t+O\left(\Delta t^{2}\right), \\
& \bar{x}_{m i}{ }^{d}(t+\Delta t)=\bar{x}_{m i}{ }^{d}(t)+\dot{\bar{x}}_{m i}{ }^{d}(t) \Delta t+O\left(\Delta t^{2}\right) .
\end{aligned}
$$

Neglecting the high-order terms of $\Delta t$ and substituting Equations (15) and(16) into Equation (14), we can obtain

$$
\begin{aligned}
\eta_{i}^{2}(t+\Delta t)-\eta_{i}^{2}(t)= & 2 x_{m i}(t) V_{x i}(t) \Delta t-2 x_{m i}(t) \dot{\bar{x}}_{m i}^{d}(t) \Delta t \\
& -2 V_{x i}(t) \bar{x}_{m i}^{d}(t) \Delta t+2 \bar{x}_{m i}^{d}(t) \dot{\bar{x}}_{m i}^{d}(t) \Delta t .
\end{aligned}
$$

Based on Equation (7), $\bar{x}_{m i}{ }^{d}(t)$ and $\dot{\bar{x}}_{m i}{ }^{d}(t)$ can be presented as

$$
\begin{aligned}
\bar{x}_{m i}{ }^{d}(t) & =x_{T}(t)+V_{T x}(t) \bar{t}_{g o i}(t), \\
\dot{\bar{x}}_{i}^{d}(t) & =V_{T x}(t)+a_{T x}(t) \bar{t}_{g o i}(t)+V_{T x}(t) \dot{\bar{t}}_{g o i}(t),
\end{aligned}
$$


where $\bar{t}_{g o i}(t)$ can be expressed as

$$
\bar{t}_{g o i}=\frac{1}{N_{i}\left(j \mid j \in N_{i}(t)\right)} \sum_{j \in N_{i}(t)} t_{g o j}(t) .
$$

From Equations (17)-(19), we have

$$
\frac{\eta_{i}^{2}(t+\Delta t)-\eta_{i}^{2}(t)}{\Delta t}=A_{i}(t)-B_{i}(t) \dot{\bar{t}}_{g o i}(t),
$$

where

$$
\begin{aligned}
A_{i}(t) & =M_{i}(t)\left(V_{x i}(t)-V_{T x}(t)-a_{T x}(t) \bar{t}_{g o i}(t)\right), \\
B_{i}(t) & =M_{i}(t) V_{T x}(t), \\
M_{i}(t) & =2\left(x_{m i}(t)-x_{T}(t)-V_{T x}(t) \bar{t}_{g o i}(t)\right) .
\end{aligned}
$$

$R_{i}(t)$ can be expressed as

$r_{i}(t)=\sqrt{\left(x_{T}(t)-x_{m i}(t)\right)^{2}+\left(y_{T}(t)-y_{m i}(t)\right)^{2}+\left(z_{T}(t)-z_{m i}(t)\right)^{2}}$.

Based on the Equations (7) and (23) and $\ddot{x}_{T}(t)-\ddot{x}_{i}(t)$ $=a_{T x}(t)-a_{i}(t)$, we have

$$
\dot{t}_{g o i}(t)=-2+\left(E_{i}(t)-\left(x_{T}(t)-x_{m i}(t)\right) a_{i}(t)\right) D_{i}(t)
$$

where

$$
\begin{gathered}
E_{i}(t)=\left(V_{T x}(t)-V_{x i}(t)\right)^{2}+\left(V_{T y}(t)-V_{y i}(t)\right)^{2} \\
+\left(y_{T}(t)-y_{m i}(t)\right)\left(a_{T y}(t)-a_{y i}(t)\right) \\
+\left(V_{T z}(t)-V_{z i}(t)\right)^{2}+\left(z_{T}(t)-z_{i}(t)\right) \\
\cdot\left(a_{T z}(t)-a_{z i}(t)\right)+\left(x_{T}(t)-x_{m i}(t)\right) a_{T x}(t), \\
D_{i}(t)=\frac{\left(x_{T}(t)-x_{m i}(t)\right)^{2}+\left(y_{T}(t)-y_{m i}(t)\right)^{2}+\left(z_{T}(t)-z_{m i}(t)\right)^{2}}{F_{i}^{2}(t)}, \\
F_{i}(t)=\left(x_{T}(t)-x_{m i}(t)\right)\left(V_{T x}(t)-V_{x i}(t)\right) \\
\quad+\left(y_{T}(t)-y_{m i}(t)\right)\left(V_{T y}(t)-V_{y i}(t)\right) \\
+\left(z_{T}(t)-z_{m i}(t)\right)\left(V_{T z}(t)-V_{z i}(t)\right) .
\end{gathered}
$$

From Equation (24), we can obtain

$$
\begin{aligned}
\dot{\bar{t}}_{g o i}(t)= & \frac{1}{\bar{N}_{i}}\left(\dot{t}_{g o i}(t)+\sum_{\substack{j \in N_{i}(t) \\
j \neq i}} \dot{t}_{g o j}(t)\right)=\frac{1}{\bar{N}_{i}}\left(-2+\left(E_{i}(t)\right.\right. \\
& \left.\left.-\left(x_{T}(t)-x_{m i}(t)\right) a_{i}(t)\right) D_{i}(t)\right)+\frac{1}{\bar{N}_{i}} \sum_{\substack{j \in N_{i}(t) \\
j \neq i}} \dot{t}_{g o j}(t),
\end{aligned}
$$

where $\bar{N}_{i}=N_{i}\left(j \mid j \in N_{i}(t)\right)$. From Equations (21) and (26), we have

$$
\begin{aligned}
\frac{\eta_{i}^{2}(t+\Delta t)-\eta_{i}^{2}(t)}{\Delta t}= & A_{i}(t)+\frac{2 B_{i}(t)}{\bar{N}_{i}}-\frac{B_{i}(t) D_{i}(t) E_{i}(t)}{\bar{N}_{i}} \\
& -\frac{1}{\bar{N}_{i}} B_{i}(t) \sum_{\substack{j \in N_{i}(t) \\
j \neq i}} \dot{t}_{g o j}(t) \\
& +\frac{B_{i}(t) D_{i}(t)\left(x_{T}(t)-x_{m i}(t)\right)}{\bar{N}_{i}} a_{i}(t) .
\end{aligned}
$$

Obviously, Equation (7) can also be rewritten as

$$
a_{i}(t)=a_{b i}(t)-P_{i}(t) a_{c i}(t),
$$

where

$$
\begin{aligned}
a_{b i}(t)= & \frac{6\left(x_{m i}^{d}(t)-x_{m i}(t)+\left(V_{T x}(t)-V_{x i}(t)\right) t_{g o i}(t)\right)}{t_{g o i}^{2}(t)} \\
& +\frac{V_{T x}\left(t_{f}\right)-V_{x i}\left(t_{f}\right)-\left(V_{T x}(t)-V_{x i}(t)\right)}{t_{g o i}(t)}+a_{T x}(t),
\end{aligned}
$$$$
a_{c i}(t)=x_{m i}{ }^{d}(t)-\bar{x}_{m i}{ }^{d}(t)=\left(t_{g o i}(t)-\bar{t}_{g o i}(t)\right) V_{T x}(t) \text {. }
$$

From Equations (27)-(29), we can obtain

$$
\begin{gathered}
\frac{\eta_{i}^{2}(t+\Delta t)-\eta_{i}^{2}(t)}{\Delta t} \\
=A_{i}(t)+\frac{2 B_{i}(t)}{\bar{N}_{i}}-\frac{B_{i}(t) D_{i}(t) E_{i}(t)}{\bar{N}_{i}}-\frac{1}{\bar{N}_{i}} B_{i}(t) \sum_{\substack{j \in N_{i}(t) \\
j \neq i}} \dot{t}_{g o j}(t) \\
+\frac{B_{i}(t) D_{i}(t)\left(x_{T}(t)-x_{m i}(t)\right)}{\bar{N}_{i}}\left(a_{b i}(t)-P_{i}(t) a_{c i}(t)\right) .
\end{gathered}
$$

When $\left(\eta_{i}^{2}(t+\Delta t)-\eta_{i}^{2}(t)\right) / \Delta t<0, \eta_{i}(t) \longrightarrow 0$, then $x_{m i}^{d}$ $(t) \longrightarrow \bar{x}_{m i}{ }^{d}(t)$, so we can have

$$
p_{i}>\frac{\mathrm{O}_{i}(t)+L_{i}(t)+W_{i}(t)}{\left|a_{c i}(t)\right| D_{i}(t)\left|M_{i}(t)\right|},
$$

where

$$
\begin{aligned}
O_{i}(t) & =\bar{N}_{i} A_{i}(t), \\
L_{i}(t) & =-B_{i}(t) \sum_{j=i, j \neq i} \dot{t}_{g o j}(t), \\
W_{i}(t) & =B_{i}(t)\left(2-D_{i}(t)\left(E_{i}(t)-\left(x_{T}(t)-x_{m i}(t)\right) a_{b i}(t)\right)\right) .
\end{aligned}
$$

As shown in Figure 1, $x_{T}(t)>x_{m i}(t)$ can be guaranteed easily in the midcourse guidance phase, and $x_{m i}(t)-x_{T}$ 
$(t)<-R_{t}<0$, where $R_{t}$ represents terminal guidance range. $V_{T x}(t) \bar{t}_{g o i}(t)$ is smaller than $\left|x_{m i}(t)-x_{T}(t)\right|$, so we can get

$$
M_{i}(t)=2\left(x_{m i}(t)-x_{T}(t)-V_{T x}(t) \bar{t}_{g o i}(t)\right)<0 .
$$

Since $V_{x i}-V_{T}-a_{T x} \bar{t}_{g o}>0$ and $V_{T x}(t)>0$, we can obtain

$$
\begin{gathered}
O_{i}(t)=\bar{N}_{i} A_{i}(t)=\bar{N}_{i} M_{i}(t)\left(V_{x i}(t)-V_{T x}(t)\right. \\
\left.-a_{T x}(t) \bar{t}_{g o i}(t)\right)<0, \\
B_{i}(t)=M_{i}(t) V_{T x}(t)<0 .
\end{gathered}
$$

Obviously, during the midcourse guidance phase, $\dot{t}_{\text {goi }}<0$. From Equation (35), we can get

$$
L_{i}(t)=-B_{i}(t) \sum_{j=i, j \neq i} \dot{t}_{g o j}(t)<0 .
$$

According to Equation (24) and $\dot{t}_{\text {goi }}<0$, we have

$$
\left(E_{i}(t)-\left(x_{T}(t)-x_{i}(t)\right) a_{i}(t)\right) D_{i}(t)<2 \text {. }
$$

From Equation (28), when $a_{c i} \longrightarrow 0, a_{i}$ and can be approximated as $a_{i} \approx a_{b i}$, so we can get

$$
\left(E_{i}(t)-\left(x_{T}(t)-x_{m i}(t)\right) a_{b i}(t)\right) D_{i}(t)<2 .
$$

From Equations (35) and (38), we can obtain

$$
W_{i}(t)=B_{i}(t)\left(2-\left(E_{i}(t)-\left(x_{T}(t)-x_{m i}(t)\right) a_{b i}(t)\right) D_{i}(t)\right)<0 .
$$

Obviously, according to the Equations (34), (36), and (39), we can get

$$
\frac{\mathrm{O}_{i}(t)+L_{i}(t)+W_{i}(t)}{\left|a_{c i}(t)\right| D_{i}(t)\left|M_{i}(t)\right|}<0
$$

Naturally, when $p_{i}>0$, it satisfies Equation (31). So when $p_{i}$ is a positive constant, then $\eta_{i}(t+\Delta t)<\eta_{i}(t)$, that means $\eta_{i}(t)$ is positive monotone decreasing function. Furthermore, $\eta_{i}(t) \longrightarrow 0$ when $t \longrightarrow \infty$, according to Equation (12), we have $x_{m i}^{d}(t) \longrightarrow \bar{x}_{m i}{ }^{d}(t)$. This completes the proof. $\square$

Remark 5. When $p_{i}$ satisfies Equation (29), Equations (7) and (8) can ensure that $x_{m i}^{d}(t) \longrightarrow \bar{x}_{m i}{ }^{d}(t)$. However, Equation (29) is more complicated and not convenient for engineering application. For medium- and long-range missiles, the speed is faster than the target (e.g., airborne warning and control system) in the midcourse guidance phase, and the acceleration of the target is smaller, so $V_{x i}-V_{T}-a_{T x}$ $\bar{t}_{g o}>0$ can be guaranteed. When $t \longrightarrow t_{m f}$, the influence of the cooperative term $a_{c i}(t)$ gradually decreases, then $a_{c i}(t)$ $\longrightarrow 0$. Therefore, Equation (29) can be simplified to Equa- tion (40), which is convenient for practical engineering applications.

Based on Theorem 4, we can see that selecting the appropriate $p_{i}$ can ensure that $x_{m i}^{d}(t) \longrightarrow \bar{x}_{m i}{ }^{d}(t)$, but in order to ensure multiple missiles to arrive at the handover area simultaneously, it still needs to ensure that $\bar{x}_{m i}{ }^{d}(t) \longrightarrow$ $\bar{x}_{m j}{ }^{d}(t)$. When there is a directed spanning tree in the communication topology, the first-order multiagent consensus protocol can ensure that $\bar{x}_{m i}{ }^{d}(t) \longrightarrow \bar{x}_{m j}{ }^{d}(t)$. However, in the actual environment, communication delay must exist and its impact cannot be ignored. Therefore, considering the communication delay, we have the following theorem and lemma.

Lemma 6 (see [35]). Suppose that $Q$ is any $n \times n$ positive definite matrix, $X$ and $Y$ are $n$-dimension vectors, then the following matrix inequality holds:

$$
2 X^{T} Y \leq X^{T} Q^{-1} X+Y^{T} Q Y .
$$

Theorem 7. Suppose that there exists a directed spanning tree in graph $G$, the adjacency matrix $A=\left(a_{i j}\right) \in R^{n \times n}$ is a constant matrix, the diagonalized matrix of its Laplace matrix $L$ responding to $A$ is $J \in R^{n \times n}$, and the communication delay $\tau$ between any two nodes $i$ and $j$. If $\tau<1 / \lambda_{\max }(L)$, then the consensus protocol with communication delay as Equation (11) can reach agreement, that is, $\bar{x}_{m i}{ }^{d}(t) \longrightarrow \bar{x}_{m j}{ }^{d}(t)$. Further, the controller as Equation (11) can make $\bar{x}_{m i}^{d}(t)$ converge into the kernel space $\left\{\left(\tau J^{2}-J\right) T\right\}$, where $T$ is a conversion matrix for the diagonalization.

Proof. Based on the graph theory, Equation (11) can also be expressed as

$$
\dot{X}(t)=-L X(t-\tau)
$$

where $X=\left[\bar{x}_{m 1}^{d}, \bar{x}_{m 2}^{d}, \cdots, \bar{x}_{m n}^{d}\right]^{T}$ and $L=\left(l_{i j}\right) \in R^{n \times n}$ is the Laplacian matrix.

Define the Lyapunov-Krasovskii function as

$V(X)=Y^{T}(t) P Y(t)+\int_{-\tau}^{0} \int_{t+\theta}^{t} X^{T}(\omega)(-L)^{T} P(-L) X(\omega) d \omega d \theta$

where $P$ is a $n \times n$ positive matrix to be determined and $Y(t)$ can be expressed as

$$
Y(t)=X(t)+\int_{t-\tau}^{t}(-L) X(\omega) d \omega
$$


From Equation (44), we have

$$
\begin{aligned}
\dot{Y}(t) & =\dot{X}(t)+(-L) X(t)-(-L) X(t-\tau) \\
& =(-L) X(t-\tau)+(-L) X(t)-(-L) X(t-\tau) \\
& =(-L) X(t) .
\end{aligned}
$$

According to the Equation (43), $\dot{V}(t)$ can be expressed as

$$
\begin{aligned}
\dot{V}(X)= & \dot{Y}^{T}(t) P Y(t)+Y^{T}(t) P \dot{Y}(t) \\
& +\frac{d}{d t}\left(\int_{-\tau}^{0} \int_{t+\theta}^{t} X^{T}(\omega)(-L)^{T} P(-L) X(\omega) d \omega d \theta\right) .
\end{aligned}
$$

From the Equation (45), we have

$$
\begin{aligned}
\dot{Y}^{T}(t) P Y(t)= & {[(-L) X(t)]^{T} P\left(X(t)+\int_{t-\tau}^{t}(-L) X(\omega) d \omega\right) } \\
= & X^{T}(t)(-L)^{T} P X(t)+X^{T}(t) \\
& \cdot(-L)^{T} P \int_{t-\tau}^{t}(-L) X(\omega) d \omega .
\end{aligned}
$$

Similarly, $Y^{T}(t) P \dot{Y}(t)$ can also be obtained. Furthermore, the third term of the right side of Equation (46) can be calculated as follows:

$$
\begin{aligned}
\frac{d}{d t}\left(\int_{-\tau}^{0} \int_{t+\theta}^{t} X^{T}(\omega)(-L)^{T} P(-L) X(\omega) d \omega d \theta\right) \\
=\int_{-\tau}^{0} \frac{d}{d t}\left(\int_{t+\theta}^{t} X^{T}(\omega)(-L)^{T} P(-L) X(\omega) d \omega\right) d \theta \\
=\int_{-\tau}^{0}\left(X^{T}(t)(-L)^{T} P(-L) X(t)\right. \\
\left.\quad-X^{T}(t+\theta)(-L)^{T} P(-L) X(t+\theta)\right) d \theta \\
=\tau\left(X^{T}(t)(-L)^{T} P(-L) X(t)\right) \\
\quad-\int_{-\tau}^{0} X^{T}(t+\theta)(-L)^{T} P(-L) X(t+\theta) d \theta
\end{aligned}
$$

Let $\theta^{\prime}=t+\theta$, Equation (48) can further be transformed

$$
\begin{aligned}
\frac{d}{d t}\left(\int_{-\tau}^{0} \int_{t+\theta}^{t} X^{T}(\omega)(-L)^{T} P(-L) X(\omega) d \omega d \theta\right) \\
=\tau\left(X^{T}(t)(-L)^{T} P(-L) X(t)\right)-\int_{t-\tau}^{t} X^{T}\left(\theta^{\prime}\right) \\
\cdot(-L)^{T} P(-L) X\left(\theta^{\prime}\right) d \theta^{\prime} .
\end{aligned}
$$

TABLe 1: Simulation initial conditions for missiles.

\begin{tabular}{lcccc}
\hline Missile & Initial position $(\mathrm{m})$ & $V_{x 0}(\mathrm{~m} / \mathrm{s})$ & $V_{y 0}(\mathrm{~m} / \mathrm{s})$ & $V_{z 0}(\mathrm{~m} / \mathrm{s})$ \\
\hline Missile1 & $(3000,11000,-2000)$ & 850 & 50 & 60 \\
Missile2 & $(1700,13000,-1000)$ & 900 & 20 & 20 \\
Missile3 & $(0,9000,500)$ & 950 & 40 & 40 \\
Missile4 & $(-2000,14000,-1500)$ & 950 & 20 & 60 \\
Missile5 & $(1000,12000,0)$ & 850 & 40 & 40 \\
\hline
\end{tabular}

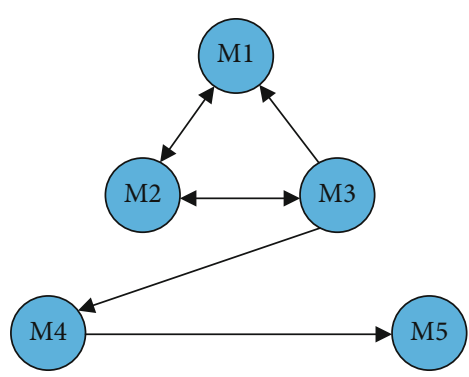

FIgURE 3: The communication topology.

Substituting Equations (47) and (49) into Equations (46), we can obtain

$$
\begin{aligned}
\dot{V}(X)= & X^{T}(t)\left[(-L)^{T} P+P L\right] X(t) \\
& +2 \int_{t-\tau}^{t} X^{T}(t)(-L)^{T} P(-L) X(\omega) d \omega \\
& +\tau\left(X^{T}(t)(-L)^{T} P(-L) X(t)\right) \\
& -\int_{t-\tau}^{t} X^{T}\left(\theta^{\prime}\right)(-L)^{T} P(-L) X\left(\theta^{\prime}\right) d \theta^{\prime}
\end{aligned}
$$

According to the Lemma 6, we have

$$
\begin{aligned}
& 2 \int_{t-\tau}^{t} X^{T}(t)(-L)^{T} P(-L) X(\omega) d \omega \\
& \quad \leq \int_{t-\tau}^{t} X^{T}(t)(-L)^{T} P Q^{-1} P(-L) X(t) d \omega \\
& \quad+\int_{t-\tau}^{t} X^{T}(\omega)(-L)^{T} Q(-L) X(\omega) d \omega
\end{aligned}
$$

where $Q$ is any appropriate dimensional positive-definite matrix. Hence, we can let $Q=P$, then Equation (51) can be expressed as

$$
\begin{aligned}
& 2 \int_{t-\tau}^{t} X^{T}(t)(-L)^{T} P(-L) X(\omega) d \omega \\
& \leq \tau\left[X^{T}(t)(-L)^{T} P(-L) X(t)\right] \\
& \quad+\int_{t-\tau}^{t} X^{T}(\omega)(-L)^{T} P(-L) X(\omega) d \omega .
\end{aligned}
$$



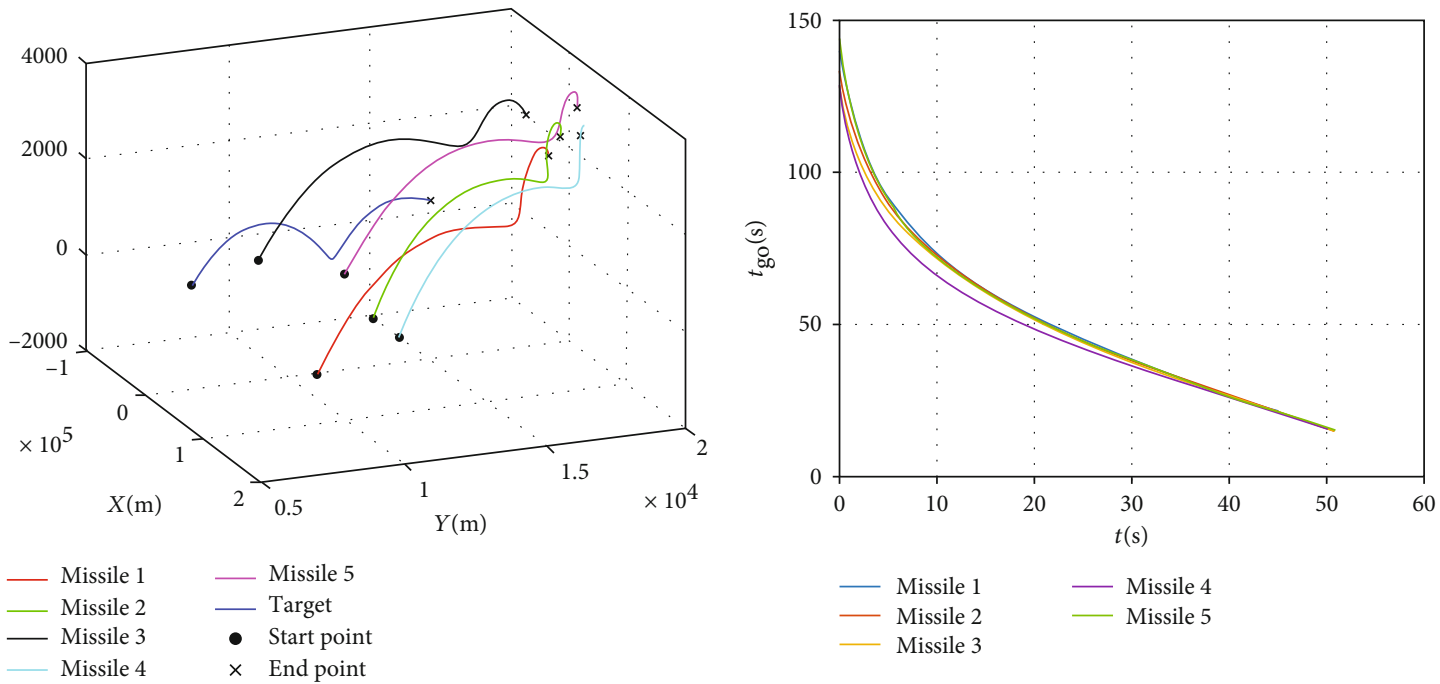

(a) The fight process without delay

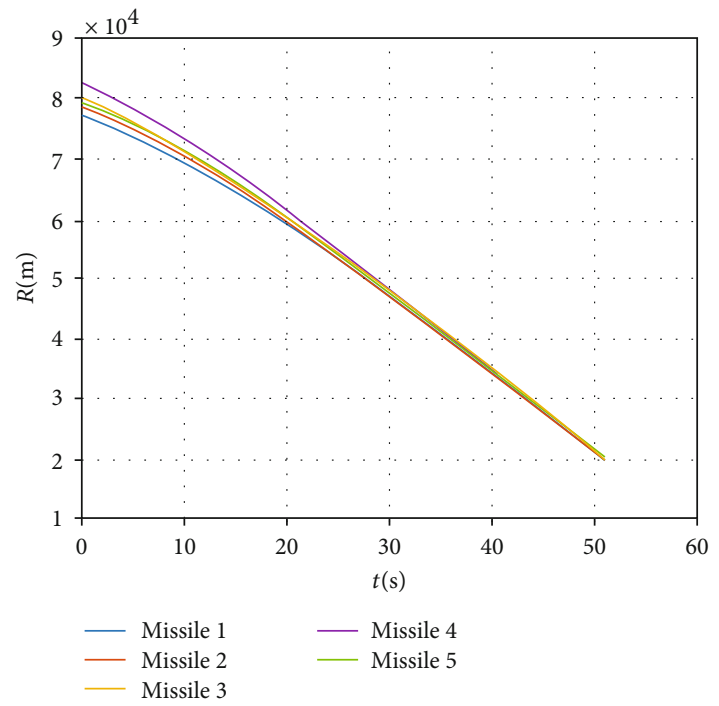

(c) Relative ranges without delay

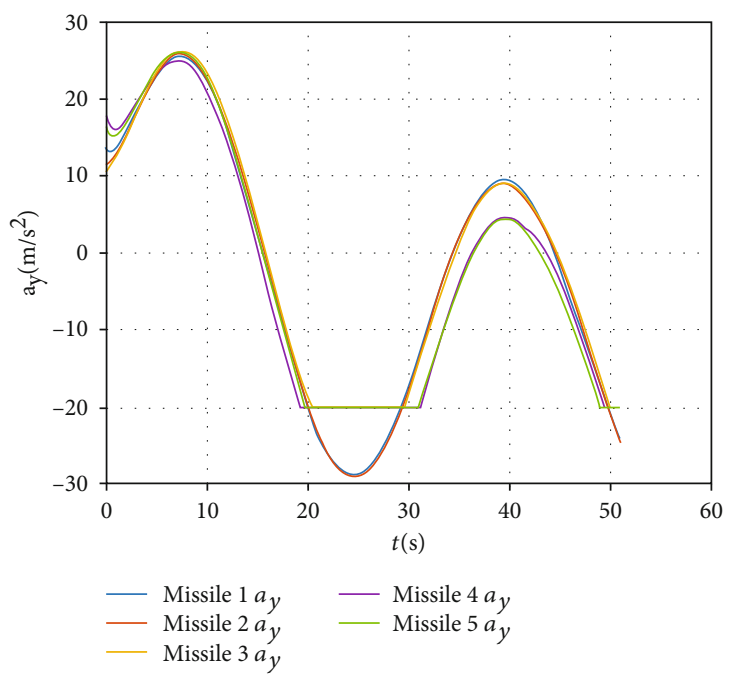

(e) The acceleration $a_{y}$ without delay

(b) Times-to-go without delay

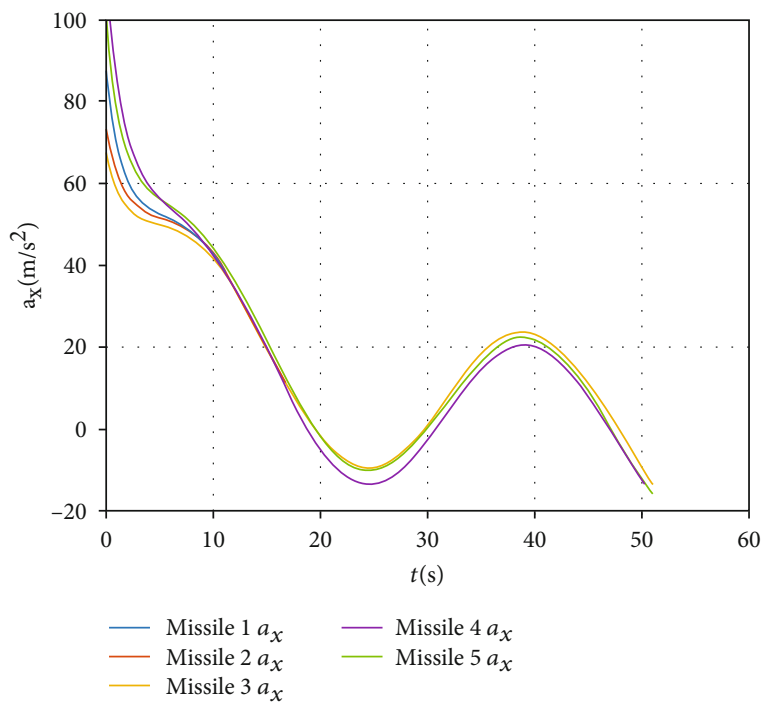

(d) The acceleration $a_{x}$ without delay

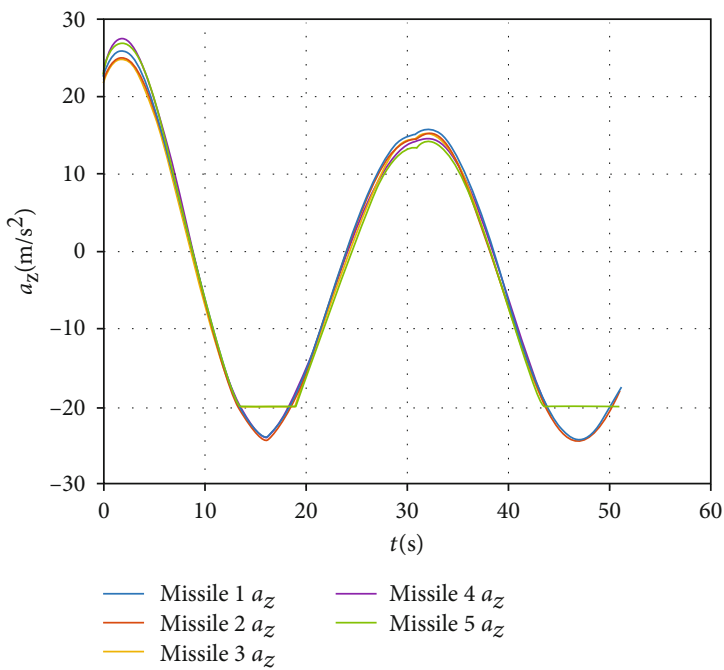

(f) The acceleration $a_{z}$ without delay

FIgURE 4: The simulation results without delay. 
TABle 2: Summary of the differences of times-to-go and relative range without delay.

\begin{tabular}{lcc}
\hline The maximum differences & Start point & End point \\
\hline The maximum difference of relative range & $5238 \mathrm{~m}$ & $470 \mathrm{~m}$ \\
The maximum difference of times-to-go & $21.20 \mathrm{~s}$ & $0.54 \mathrm{~s}$ \\
\hline
\end{tabular}

From Equations (50) and (52), we can obtain

$$
\begin{aligned}
\dot{V}(X) \leq & X^{T}(t)\left[(-L)^{T} P+P(-L)\right] X(t) \\
& +\tau\left[X^{T}(t)(-L)^{T} P(-L) X(t)\right] \\
& +\int_{t-\tau}^{t} X^{T}(\omega)(-L)^{T} P(-L) X(\omega) d \omega \\
& +\tau\left(X^{T}(t)(-L)^{T} P(-L) X(t)\right) \\
& -\int_{t-\tau}^{t} X^{T}\left(\theta^{\prime}\right)(-L)^{T} P(-L) X\left(\theta^{\prime}\right) d \theta^{\prime} \\
= & X^{T}(t) Z X(t),
\end{aligned}
$$

where

$$
Z=(-L)^{T} P+P(-L)+2 \tau(-L)^{T} P(-L) .
$$

Since the Laplacian matrix $L$ is a diagonalizable matrix, so $L$ can be expressed as $L=T^{-1} J T$, where $J \in R^{n \times n}$ is a diagonal matrix composed of the eigenvalues of the Laplacian matrix $L$. Because $P$ is a positive matrix to be determined, we can let $P=T^{T} T$, and substituting it into Equation (53), we can further calculate $\dot{V}(X)$ as

$$
\begin{aligned}
\dot{V}(X) \leq & X^{T}(t)\left[\left(-T^{-1} J T\right)^{T}\left(T^{T} T\right)+\left(T^{T} T\right)\left(-T^{-1} J T\right)\right. \\
& \left.+2 \tau\left(-T^{-1} J T\right)^{T}\left(T^{T} T\right)\left(-T^{-1} J T\right)\right] X(t) \\
= & X^{T}(t)\left[-T^{T} J T^{-T} T^{T} T-T^{T} T T^{-1} J T\right. \\
& \left.+2 \tau T^{T} J T^{-T} T^{T} T T^{-1} J T\right] X(t) \\
= & X^{T}(t)\left[-T^{T} J T-T^{T} J T+2 \tau T^{T} J^{2} T\right] X(t) \\
= & 2(T X(t))^{T}\left[-J+\tau J^{2}\right] T X(t) .
\end{aligned}
$$

Since the Laplacian matrix $L$ is a positive semidefinite matrix and has a eigenvalue is 0 , then diagonal matrix $J \in$ $R^{n \times n}$ can be expressed as

$$
J=\left[\begin{array}{llll}
0 & & & \\
& \lambda_{2}(L) & & \\
& & \ddots & \\
& & & \lambda_{n}(L)
\end{array}\right]^{n \times n},
$$

then, we have

$$
-J+\tau J^{2}=\left[\begin{array}{llll}
0 & & & \\
& -\lambda_{2}(L) & & \\
& & \ddots & \\
& & -\lambda_{n}(L)
\end{array}\right]
$$

$$
+\left[\begin{array}{llll}
0 & & & \\
& \tau \lambda_{2}^{2}(L) & & \\
& & \ddots & \\
& & & \tau \lambda_{n}^{2}(L)
\end{array}\right]
$$

$$
=\left[\begin{array}{llll}
0 & & & \\
& \tau \lambda_{2}^{2}(L)-\lambda_{2}(L) & & \\
& & \ddots & \\
& & & \tau \lambda_{n}^{2}(L)-\lambda_{n}(L)
\end{array}\right] .
$$

Since $\lambda_{i}(L)>0(i=2, \cdots, n)$, if $\tau<1 / \lambda_{\max }(L)$, we have

$$
\tau \lambda_{i}^{2}(L)-\lambda_{i}(L)<0 \quad i=2, \cdots, n .
$$

From Equations (55) and (58), we can obtain

$$
\dot{V}(X) \leq 2(T X(t))^{T}\left[-J+\tau J^{2}\right] T X(t) \leq 0 .
$$

According to the Lasselle invariant set theorem, the state of the system as Equation (42) converges into the set $\Omega=$ $\left\{X \in R^{n} \mid \dot{V}(X)=0\right\}$. In the following, we will divide the inequality as Equality (59) into three cases to prove the system as Equality (11) to reach an agreement.

Case 1. When $(T X(t))^{T}\left[-J+\tau J^{2}\right] T X(t)<0$, then $\dot{V}(X)<0$. According to the Lyapunov stability theorem, the system as Equation (42) is stable, which means the system as Equation (11) is stable. Hence, the protocol can ensure the multiagent system as Equation (11) to reach an agreement.

Case 2. When $(T X(t))^{T}\left[-J+\tau J^{2}\right] T X(t)=0$, but $\dot{V}(X)<2$ $(T X(t))^{T}\left[-J+\tau J^{2}\right] T X(t)$, then $\dot{V}(X)<0$, similar to Case 1 , we can prove that the protocol can ensure the multiagent system as Equation (11) to reach an agreement.

Case 3. When $(T X(t))^{T}\left[-J+\tau J^{2}\right] T X(t)=0$ and $\dot{V}(X)=2$ $(T X(t))^{T}\left[-J+\tau J^{2}\right] T X(t)$, then $\dot{V}(X)=0$. According to the Lasselle invariant set theorem, the state of the system as Equation (42) converges into the set $\Omega=\left\{X \in R^{n} \mid \dot{V}(X)=\right.$ $0\}=\left\{X \mid(T X(t))^{T}\left[-J+\tau J^{2}\right] T X(t)=0\right\} .$. 


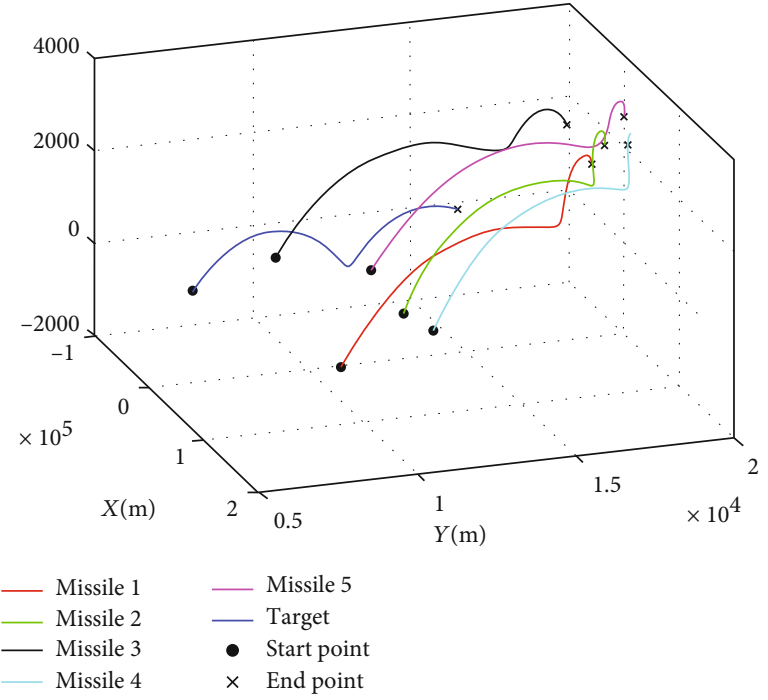

(a) The fight process with $\tau=0.2 \mathrm{~s}$
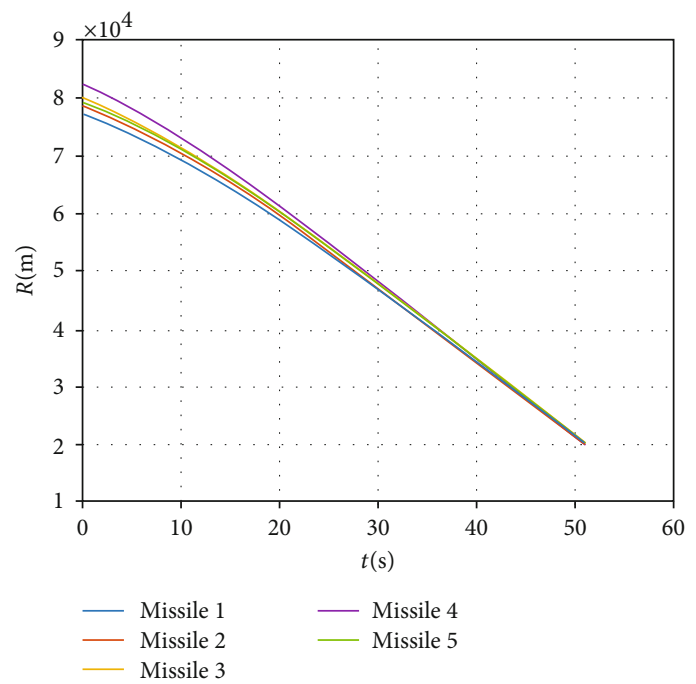

(c) Relative ranges with $\tau=0.2 \mathrm{~s}$

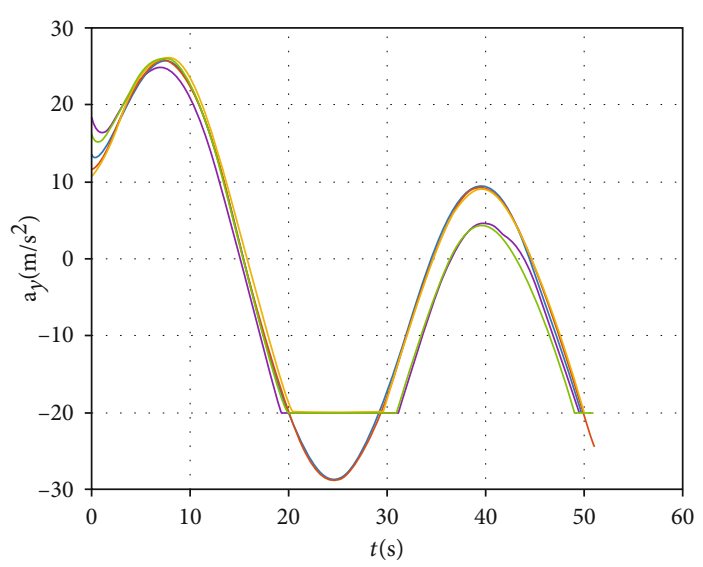

$\begin{array}{rr}\text { Missile } 1 \mathrm{a}_{y} & \text { - Missile } 4 \mathrm{a}_{y} \\ \text { Missile } 2 \mathrm{a}_{y} & \text { - Missile } 5 \mathrm{a}_{y}\end{array}$

(e) The acceleration $a_{y}$ with $\tau=0.2 \mathrm{~s}$

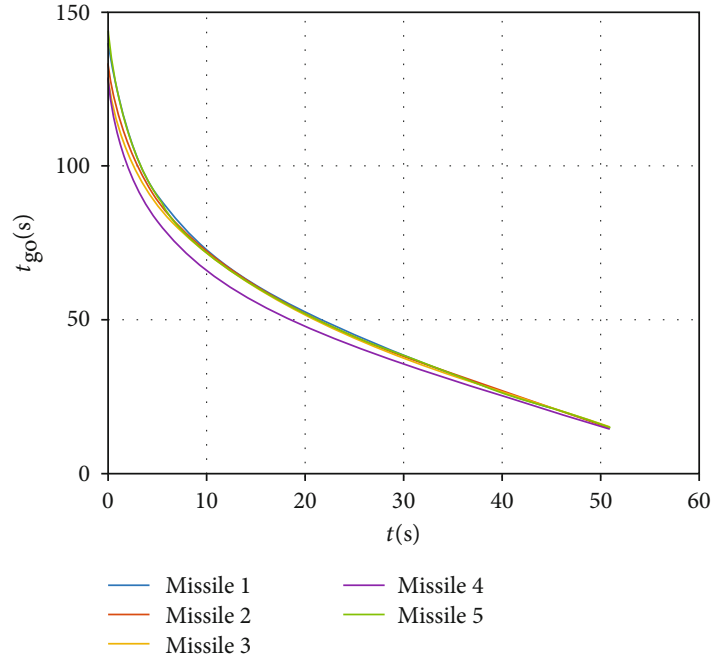

(b) Times-to-go with $\tau=0.2 \mathrm{~s}$

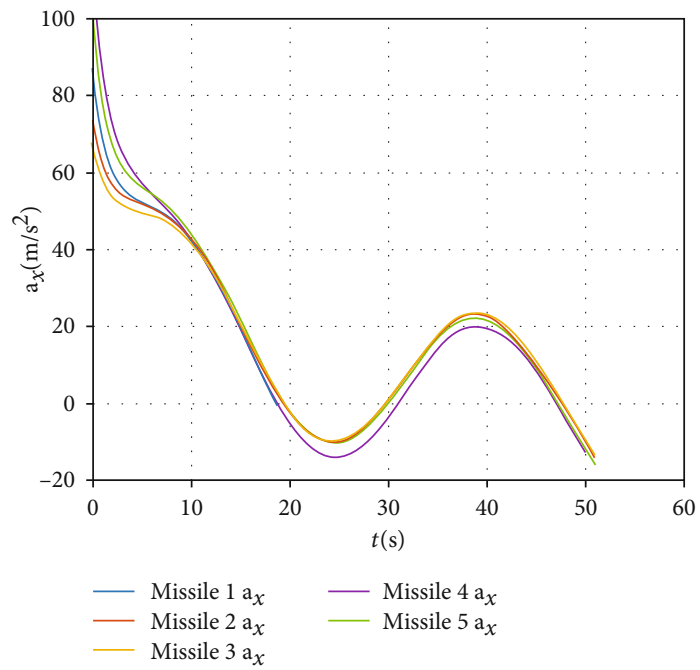

(d) The acceleration $a_{x}$ with $\tau=0.2 \mathrm{~s}$

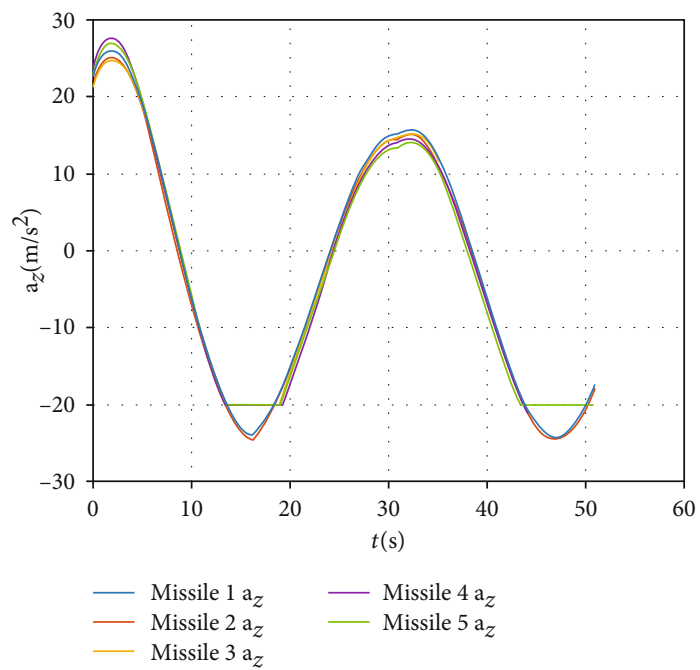

(f) The acceleration $a_{z}$ with $\tau=0.2 \mathrm{~s}$

FIgURE 5: The simulation results with delay $\tau=0.2 \mathrm{~s}$. 
TABLE 3: Summary of the differences of times-to-go and relative range with delay $\tau=0.2 \mathrm{~s}$.

\begin{tabular}{lcc}
\hline The maximum differences & Start point & End point \\
\hline The maximum difference of relative range & $5238 \mathrm{~m}$ & $512 \mathrm{~m}$ \\
The maximum difference of times-to-go & $21.20 \mathrm{~s}$ & $0.71 \mathrm{~s}$ \\
\hline
\end{tabular}

Denote the kernel space as ker $\left\{\left(-J+\tau J^{2}\right) T\right\}=\{X \mid(-$ $\left.\left.J+\tau J^{2}\right) T X=0\right\}$. Obviously, $\operatorname{ker}\left\{\left(-J+\tau J^{2}\right) T\right\}=\Omega$. From Equation (57), we know that $-J+\tau J^{2}$ has an eigenvalue $\lambda_{1}$ $\left(-J+\tau J^{2}\right)=0$, and other eigenvalues $\lambda_{i}\left(-J+\tau J^{2}\right) \neq 0$ $(i=2, \cdots, n)$, so $\operatorname{rank}\left\{\left(-J+\tau J^{2}\right) T\right\}=1$, then $\operatorname{ker}\{(-J+\tau$ $\left.\left.J^{2}\right) T\right\}$ is a one-dimensional subspace. Furthermore, we can have

$$
\left(-J+\tau J^{2}\right) T T^{-1}\left(\left[\begin{array}{c}
1 \\
0 \\
\vdots \\
0
\end{array}\right] \otimes \zeta\right)=0
$$

where $\zeta \in R$. According to Equation (60), we can obtain

$$
\operatorname{span}\{\Pi\} \subseteq \operatorname{ker}\left(-J+\tau J^{2}\right),
$$

where

$$
\operatorname{span}\{\Pi\}=\operatorname{span}\left\{T^{-1}\left(\left[\begin{array}{c}
1 \\
0 \\
\vdots \\
0
\end{array}\right] \otimes \zeta\right)\right\}
$$

On the other hand, $\operatorname{span}\{\Pi\}$ is a one-dimensional subspace, so we can have

$$
\operatorname{span}\{\Pi\}=\operatorname{ker}\left(-J+\tau J^{2}\right) .
$$

This completes the proof.

Remark 8. In Refs. [29, 31], the communication topology is required to be an undirected connected graph. However, in this paper, the proposed consensus protocol with communication delay as Equation (11)) requires the existence of a directed spanning tree in the communication topology and has lower requirements for communication resources than the undirected graph. Furthermore, the method proposed in this paper can directly obtain the upper bound of the communication delay through the communication topology, which is simpler and more conducive to practical engineering applications than the LMI method.

As can be seen from Theorems 4 and 7, the proposed DCMG law with communication delay as Equation (12) ensures that multiple missiles reach the same virtual target collision point. In the following, we will prove the time con- sistency of the proposed DCMG law with communication delay.

Theorem 9. Suppose that the conditions are same as Theorem 7 , if $\bar{x}_{m i}{ }^{d}(t) \longrightarrow \bar{x}_{m j}^{d}(t)$, then $\bar{t}_{g o i}(t)-\bar{t}_{g o j}(t) \longrightarrow 0$.

Proof. From Equations (7) and (9), we can obtain

$$
\bar{x}_{m i}^{d}(t)=x_{T}(t)+V_{T x}(t) \bar{t}_{g o i}(t) .
$$

According to Equation (64), we can get

$$
\bar{t}_{g o i}(t)=\frac{\bar{x}_{m i}^{d}(t)-x_{T}(t)}{V_{T x}(t)} .
$$

So, $\bar{t}_{g o i}(t)-\bar{t}_{g o j}(t)$ can be expressed as

$$
\begin{aligned}
\bar{t}_{g o i}(t)-\bar{t}_{g o j}(t) & =\frac{\bar{x}_{m i}^{d}(t)-x_{T}(t)}{V_{T x}(t)}-\frac{\bar{x}_{m j}^{d}(t)-x_{T}(t)}{V_{T x}(t)} \\
& =\frac{\bar{x}_{m i}^{d}(t)-\bar{x}_{m j}^{d}(t)}{V_{T x}(t)} .
\end{aligned}
$$

According to Theorem 7, when $t \longrightarrow \infty$, we can obtain $\bar{x}_{m i}^{d}(t)-\bar{x}_{m j}^{d}(t) \longrightarrow 0$. Therefore, from Equation (66), we can get $\bar{t}_{g o i}(t)-\bar{t}_{g o j}(t) \longrightarrow 0$. This completes the proof. $\square$

Based on the discussion and analysis of the above theorems, we can obtain Theorem 10 .

Theorem 10. Suppose that the conditions are same as Theorem 7 , the proposed DCMG with communication delay is given as Equation (12) and $p_{i}>0$; then, all missiles can arrive at the mid-to-terminal handover area simultaneously.

Proof. According to Theorem 4, when $p_{i}>0$, the proposed DCMG law can ensure that $x_{m i}^{d}(t) \longrightarrow \bar{x}_{m i}{ }^{d}(t)$. Due to the directed graph $G$ including a directed spanning tree, we can obtain $\bar{x}_{m i}{ }^{d}(t) \longrightarrow \bar{x}_{m j}{ }^{d}(t)$ with communication delay from Theorem 7. Furthermore, according to Theorem 9, we can obtain $\bar{t}_{g o i}(t)-\bar{t}_{g o j}(t) \longrightarrow 0$ when $t \longrightarrow \infty$.So from Theorems 4-9, the proposed DCMG law with communication delay can ensure that $\left(x_{m i}^{d}(t)-x_{m j}^{d}(t)\right) \longrightarrow\left(\bar{x}_{m i}{ }^{d}(t)-\right.$ $\left.\bar{x}_{m j}{ }^{d}(t)\right) \longrightarrow\left(\bar{t}_{g o i}(t)-\bar{t}_{g o j}(t)\right) \longrightarrow 0$, which presents multimissile arrive at the mid-to-end handover area simultaneously. This completes the proof. $\square$

\section{Simulation}

In this section, the performance of the proposed DCMG law with communication delay is verified through numerical simulations. Considering the situation of which five missiles cooperate to strike the same target, three cases simulation experiments were carried out. In Case 1, there is no communication delay between missiles. In Case 2 and Case 3, the communication delays are different; one is lower than the 

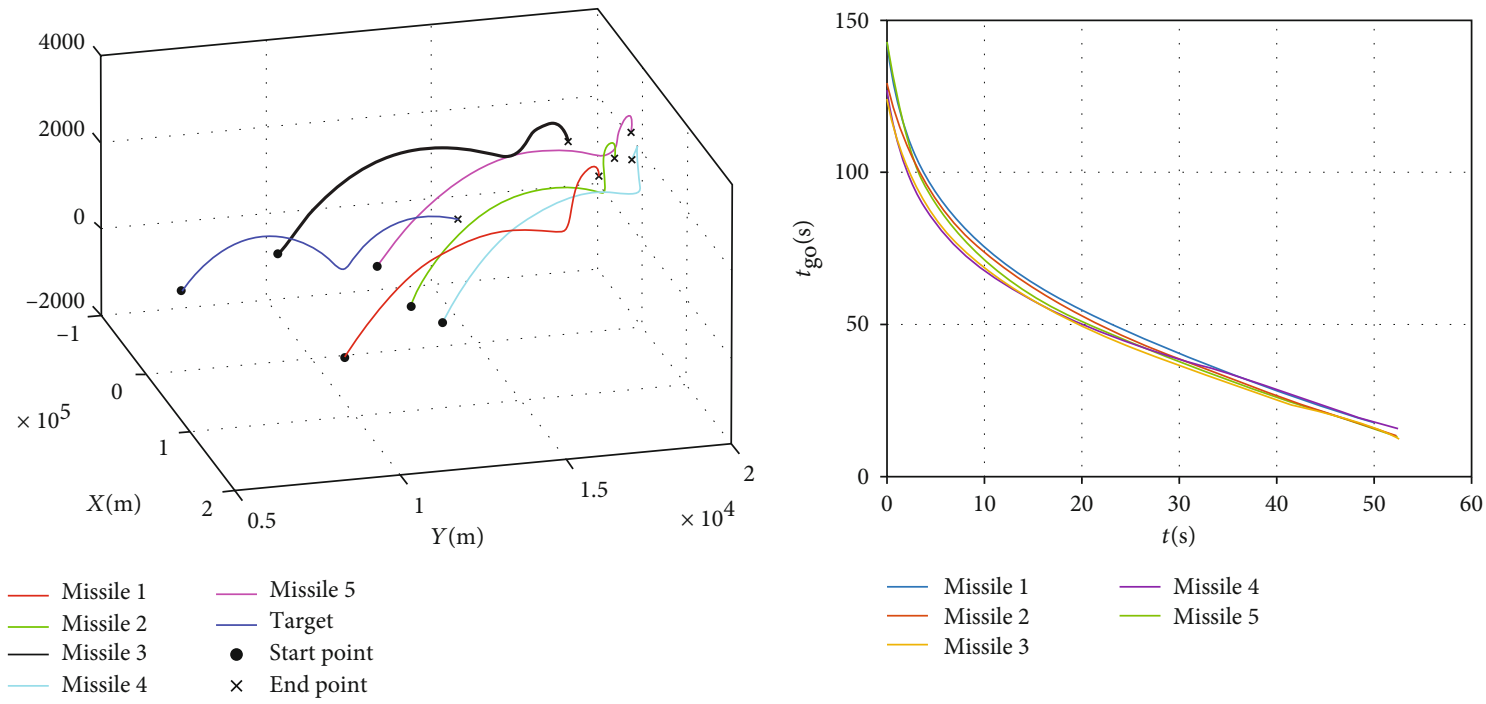

(a) The fight process with $\tau=0.4 \mathrm{~s}$
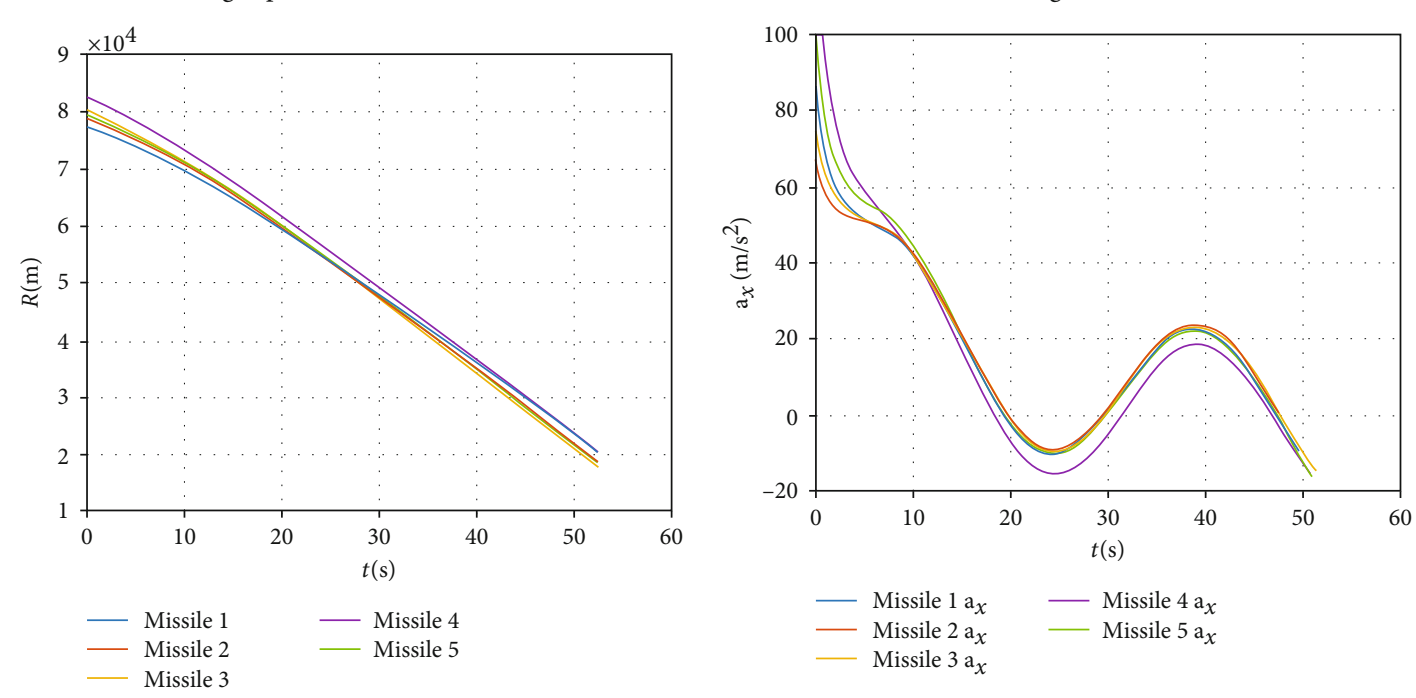

(c) Relative ranges with $\tau=0.4 \mathrm{~s}$

(d) The acceleration $a_{x}$ with $\tau=0.4 \mathrm{~s}$

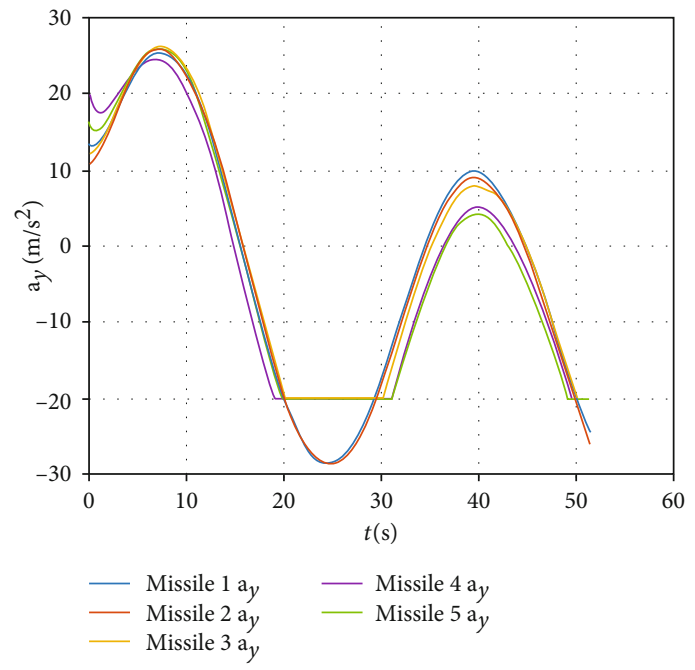

(e) The acceleration $a_{y}$ with $\tau=0.4 \mathrm{~s}$

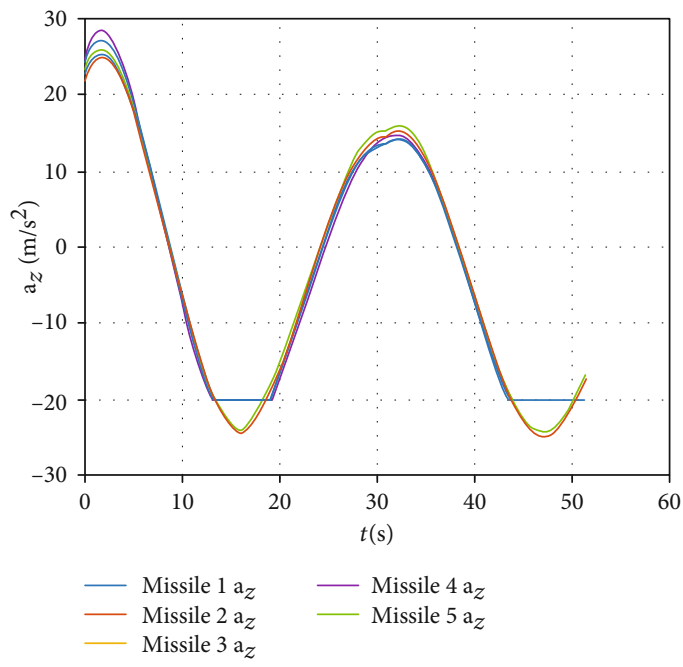

(f) The acceleration $a_{z}$ with $\tau=0.4 \mathrm{~s}$

Figure 6: The simulation results with delay $\tau=0.4 \mathrm{~s}$. 
TABLE 4: Summary of the differences of times-to-go and relative range with delay $\tau=0.4 \mathrm{~s}$.

\begin{tabular}{lcc}
\hline The maximum differences & Start point & End point \\
\hline The maximum difference of relative range & $5238 \mathrm{~m}$ & $1201 \mathrm{~m}$ \\
The maximum difference of times-to-go & $21.20 \mathrm{~s}$ & $1.32 \mathrm{~s}$ \\
\hline
\end{tabular}

upper bound of the communication delay, and another is greater than the upper bound of the communication delay. The initial location and speed of the target are 80000, 5000 , and $1000 \mathrm{~m}$ and 300,20 , and $10 \mathrm{~m} / \mathrm{s}$, respectively. Assuming when the distance from the missile to the target reaches $20 \mathrm{~km}$, the midcourse guidance phase will terminate. The initial conditions of missiles are shown in Table 1, and the target is supposed to be a maneuvering target with $a_{T}=(20 \sin (0.2 t), 20 \sin (0.2 t), 20 \sin (0.2 t+$ $1.57075)$ ), respectively. The directed communication topology is shown in Figure 3; the upper bound of the communication delay can be obtained as $\tau^{*}=0.33 \mathrm{~s}$ from Theorem 7 . In the cooperative midcourse guidance phase, the acceleration commands of the missiles are restricted as $\left|a_{i}\right|<10 \mathrm{~g}$, $i=1,2, \cdots, n$. Since the speed of the missile is fast in the midcourse guidance phase (greater than $1000 \mathrm{~m} / \mathrm{s}$ ), it is assumed that at the end of the midcourse guidance phase, the maximum difference of relative ranges is less than $1 \mathrm{~km}\left(\sigma_{1}=\right.$ $1000 \mathrm{~m}$ ) and the maximum difference of times-to-go is less than $1 \mathrm{~s}\left(\sigma_{2}=1 \mathrm{~s}\right)$, which meets the requirements of the cooperative midcourse guidance phase. In the following simulation experiment, the delay caused by the actuator is considered, so the acceleration command can be expressed as

$$
\dot{a}_{r t}(t)=-\frac{1}{T} a_{r t}(t)+\frac{1}{T} a_{i}(t)
$$

where $a_{r t}(t)$ is the actual acceleration command and $T$ is the time constant and setting as $T=0.1 \mathrm{~s}$.

4.1. Case 1 (without Communication Delay). The simulation results, including the fight trajectory, the relative ranges between the missiles and the target, the times-to-go of missiles, and the accelerations of missiles, are depicted in Figures 4(a)-4(f). The proposed DCMG without communication delay can ensure that all missiles almost simultaneously arrive at the handover area about $20 \mathrm{~km}$ from the target seen in Figures 4(a)-4(c). Furthermore, it can be seen from Figure 4 that due to the influence of the virtual collision point, at the end of the midcourse guidance phase, all the missiles form a surrounding situation on the target, which provides good initial conditions for the terminal guidance phase. From Figures 4(b) and 4(c), it can be seen that the times-to-go and the relative ranges of missiles converge gradually. As can be seen from Table 2, the maximum differences of relative ranges and times-to-go gradually decrease from $5238 \mathrm{~m}$ to $470 \mathrm{~m}$ and $21.20 \mathrm{~s}$ to $0.54 \mathrm{~s}$, respectively, which meets the requirements of the cooperative midguidance phase. According to Figures 4(d)-4(f), in order to achieve consistency, the acceleration commands of all missiles are larger in the initial phase.
4.2. Case 2 (with Communication Delay $\tau=0.2 s$ ). The simulation results, including the fight trajectory, the relative range between the missile and the target, the times-to-go of missiles, and the accelerations of missiles, are depicted in Figures 5(a)-5(f). Because the selected communication delay is less than its upper bound, the proposed DCMG with communication delay can ensure that all missiles almost simultaneously arrive at the handover area about $20 \mathrm{~km}$ from the target shown in Figures 5(a)-5(c). Furthermore, it can be seen from Figure 5(a) that due to the influence of the virtual collision point, at the end of the midcourse guidance phase, all the missiles form a surrounding situation on the target, which provides good initial conditions for the terminal guidance phase. From Figures 5(b) and 5(c), it can be seen that the times-to-go and the relative ranges of missiles converge gradually. As can be seen from Table 3, the maximum differences of relative ranges and times-to-go gradually decrease from $5238 \mathrm{~m}$ to $512 \mathrm{~m}$ and $21.20 \mathrm{~s}$ to $0.71 \mathrm{~s}$, respectively, which meets the requirements of the cooperative midguidance phase. Through Tables 2 and 3, the proposed DCMG law with communication delay can not only meet the requirements of the mid-course guidance section but also the maximum differences of relative ranges and times-to-go of missiles in Case 2 are almost the same as in the Case 1 without communication delay. Comparing Figures 4(d)-4(f) with Figures 5(d)-5(f), it can be seen that in order to overcome the influence of communication delay, the acceleration command with communication delay in Case 2 is larger than in Case 1.

4.3. Case 2 (with Communication Delay $\tau=0.4$ s). The simulation results, including the fight trajectory, the relative range between the missile and the target, the times-to-go of missiles, and the accelerations of missiles, are depicted in Figures 6(a)-6(f). From Figures 6(b) and 6(c), it can be seen that the differences of times-to-go and the relative ranges of missiles are significantly larger. As can be seen from Table 4, the maximum differences of relative ranges and times-to-go gradually decrease from $5238 \mathrm{~m}$ to $1201 \mathrm{~m}$ and $21.20 \mathrm{~s}$ to $1.32 \mathrm{~s}$, respectively, which cannot meet the requirements of the cooperative midguidance phase. Through Tables 3 and 4, the proposed DCMG law with communication delay cannot meet the requirements of the cooperative midguidance phase when the communication delay exceeds the upper bound. Comparing Figures 4(d)-4(f) with Figures $6(d)-6(f)$, it can be seen that in order to overcome the influence of communication delay, the acceleration command with communication delay in Case 3 is larger than that in Case 1, but not enough to meet the requirements of the cooperative midcourse guidance phase.

\section{Conclusions}

In order to provide better initial conditions for the cooperative terminal guidance phase, a DCMG law with communication delay against a maneuvering target was proposed by combining the trajectory shaping guidance with the cooperative term based on the distributed consensus protocol with communication delay. Then, the time-space consensus of 
the proposed DCMG law with communication delay was proved. Furthermore, the upper bound of the communication delay was calculated based on communication topology. Finally, it is verified by simulation experiments that the proposed DCMG law with communication delay in this paper can effectively compensate for the influence of a range of communication delay.

\section{Data Availability}

No data were used to support this study.

\section{Conflicts of Interest}

The authors declare that they have no conflicts of interest.

\section{Acknowledgments}

The authors are grateful for the support provided by the National Natural Science Foundation of China (Grant No. 61973253), the Aerospace Science Foundation of China (20180153001), and the Foundation of National Science and Technology Key Laboratory (6142219180202).

\section{References}

[1] in-Soo Jeon, Jin-Ik Lee, and Min-Jea Tahk, "Impact-time-control guidance law for anti-ship missiles," IEEE Transactions on control systems technology, vol. 14, no. 2, pp. 260-266, 2006.

[2] Jin-Ik Lee, in-Soo Jeon, and Min-Jea Tahk, "Guidance law to control impact time and angle," IEEE Transactions on Aerospace and Electronic Systems, vol. 43, no. 1, pp. 301-310, 2007.

[3] Y. A. Zhang, G. X. Ma, and A. L. Liu, "Guidance law with impact time and impact angle constraints," Chinese Journal of Aeronautics, vol. 26, no. 4, pp. 960-966, 2013.

[4] X. F. Wang, Y. Y. Zheng, and H. Lin, "Integrated guidance and control law for cooperative attack of multiple missiles," Aerospace Science and Technology, vol. 42, pp. 1-11, 2015.

[5] I. S. Jeon, J. I. Lee, and M. J. Tahk, "Homing guidance law for cooperative attack of multiple missiles," Journal of Guidance Control Dynamics, vol. 33, no. 1, pp. 275-280, 2010.

[6] G. A. Harrison, "Hybrid guidance law for approach angle and time-of-arrival control," Journal of Guidance, Control, and Dynamics, vol. 35, no. 4, pp. 1104-1114, 2012.

[7] X. L. Wang, Y. A. Zhang, and H. L. Wu, "Distributed cooperative guidance of multiple anti-ship missiles with arbitrary impact angle constraint," Aerospace Science and Technology, vol. 46, pp. 299-311, 2015.

[8] J. Zhou and J. Yang, "Guidance law design for impact time attack against moving targets," IEEE Transactions on Aerospace and Electronic Systems, vol. 54, no. 5, pp. 2580-2589, 2018.

[9] J. L. Zhou and Y. Lu, "Cooperative guidance law design for simultaneous attack with multiple missiles against a maneuvering target," Journal of the System Science, vol. 31, pp. 287301, 2018.

[10] J. L. Zhou and J. Y. Yang, "Distributed guidance law design for cooperative simultaneous attacks with multiple missiles," Journal of Guidance, Control, and Dynamics, vol. 39, no. 10, pp. 2439-2447, 2016.
[11] D. L. Hou, Q. Wang, X. J. Sun, and C. Y. Dong, "Finite-time cooperative guidance laws for multiple missiles with acceleration saturation constraints," IET Control Theory \& Applications, vol. 9, no. 10, pp. 1525-1535, 2015.

[12] S. Zhang, Y. Guo, Z. Liu, S. Wang, and X. Hu, "Finite-time cooperative guidance strategy for impact angle and time control," IEEE Transactions on Aerospace and Electronic Systems, vol. 57, no. 2, pp. 806-819, 2021.

[13] J. Zhao, R. Zhou, and Z. N. Dong, "Three-dimensional cooperative guidance laws against stationary and maneuvering targets," Chinese Journal of Aeronautics, vol. 28, no. 4, pp. 1104-1120, 2015.

[14] J. Zhao and R. Zhou, "Distributed three-dimensional cooperative guidance via receding horizon control," Chinese Journal of Aeronautics, vol. 29, no. 4, pp. 972-983, 2016.

[15] J. H. Song, S. M. Song, and S. L. Xu, “Three-dimensional cooperative guidance law for multiple missiles with finite- time convergence," Aerospace Science and Technology, vol. 67, pp. 193-205, 2017.

[16] T. Zhang and J. Yang, "Cooperative guidance for simultaneous attack: a fully distributed, adaptive, and optimal approach," International Journal of Control, vol. 93, no. 8, pp. 17651774, 2020.

[17] S. Zhai, X. Wei, and J. Yang, "Cooperative guidance law based on time-varying terminal sliding mode for maneuvering target with unknown uncertainty in simultaneous attack," Journal of the Franklin Institute, vol. 357, no. 16, pp. 1191411938, 2020.

[18] T. Lyu, C. Li, Y. Guo, and G. Ma, “Three-dimensional finitetime cooperative guidance for multiple missiles without radial velocity measurements," Chinese Journal of Aeronautics, vol. 32, no. 5, pp. 1294-1304, 2019.

[19] L. Song, Y. A. Zhang, D. Huang, and S. Fu, "Cooperative simultaneous attack of multi-missiles under unreliable and noisy communication network: a consensus scheme of impact time," Aerospace Science and Technology, vol. 47, pp. 31-41, 2015.

[20] X. H. Wang and X. Lu, "Three-dimensional impact angle constrained distributed guidance law design for cooperative attacks," ISA Transactions, vol. 73, pp. 79-90, 2018.

[21] B. Yang, W. Jing, and C. Gao, "Three-dimensional cooperative guidance law for multiple missiles with impact angle constraint," Journal of Systems Engineering and Electronics, vol. 31, no. 6, pp. 1286-1296, 2020.

[22] Y. A. Zhang, X. L. Wang, and H. L. Wu, "A distributed cooperative guidance law for salvo attack of multiple anti-ship missiles," Chinese Journal of Aeronautics, vol. 28, no. 5, pp. 1438$1450,2015$.

[23] Z. Chen, W. Chen, X. Liu, and J. Cheng, "Three-dimensional fixed-time robust cooperative guidance law for simultaneous attack with impact angle constraint," Aerospace Science and Technology, vol. 110, article 106523, 2021.

[24] Y. Chen, J. Wang, J. Shan, and M. Xin, "Cooperative guidance for multiple powered missiles with constrained impact and bounded speed," Journal of Guidance, Control, and Dynamics, vol. 44, no. 4, pp. 825-841, 2021.

[25] J. Zhou, Y. Lv, G. Wen, and G. Chen, “Terminal-time synchronization of multivehicle systems under sampled-data communications," IEEE Transactions on Systems, Man, and Cybernetics: Systems, vol. 99, pp. 1-12, 2021.

[26] J. Zhou, X. Wu, Y. Lv, and G. Wen, “Terminal-time synchronization of multiple vehicles under discrete-time 
communication networks with directed switching topologies," IEEE Transactions on Circuits and Systems II: Express Briefs, vol. 67, no. 11, pp. 2532-2536, 2020.

[27] J. Zeng, L. H. Dou, and B. Xin, "A joint mid-course and terminal course cooperative guidance law for multi- missile salvo attack," Chinese Journal of Aeronautics, vol. 31, no. 6, pp. 1311-1326, 2018.

[28] Z. Wu, Y. Fang, W. Fu, Z. Wang, and W. Ma, “Three-dimensional cooperative mid-course guidance law against the maneuvering target," IEEE Access, vol. 8, pp. 18841-18851, 2020.

[29] S. M. He, M. Kim, T. Song, and D. Lin, "Three-dimensional salvo attack guidance considering communication delay," Aerospace Science and Technology, vol. 73, pp. 1-9, 2018.

[30] L. Zhaohui, L. Yuezu, and Z. Jialing, "Cooperative guidance law design on simultaneous attack for multiple missiles under time-delayed communication topologies," in IEEE Symposium Series on Computational Intelligence conference, pp. 20062011, Xiamen, China, 2019.

[31] G. F. Li, Y. Wu, and P. Xu, "Fixed-time cooperative guidance law with input delay for simultaneous arrival," International Journal of Control, vol. 94, no. 6, pp. 1664-1673, 2021.

[32] C. Y. Zhang, J. M. Song, and L. Huang, "The time-to-go consensus of multi-missiles with communication delay," in the 36th Chinese Control Conference, pp. 7634-7638, Dalian, China, July 26-28, 2017.

[33] S. Yi, X. Y. She, D. Guo, T. Zhao, C. W. Yang, and J. Li, "Distributed multi-munition cooperative guidance based on clock synchronization for switching and noisy networks," Journal of supercomputing, vol. 77, no. 1, article 3244, pp. 212-243, 2021.

[34] A. John, I. V. Lukacs, and O. A. Yakimenko, "Trajectoryshape-varying missile guidance for interception of ballistic missiles during the boost phase," in the AIAA Guidance, Navigation and Control Conference and Exhibit, Hilton head, South Carolina, 2007.

[35] G. H. Golub and C. F. Van Loan, Matrix Computations, The Johns Hopkins University Press, 4th ed edition, 2013. 